\title{
Climatic niche comparison across a cryptic species complex
}

\author{
Qing Zhao ${ }^{\text {Equal first author, 1 }}$, Hufang Zhang ${ }^{\text {Equal first author, 2 }}$, Jiufeng Wei ${ }^{\text {Corresp. } 1}$ \\ ${ }^{1}$ Department of Entomology, Shanxi Agricultural University, Taigu, Shanxi, P. R. China \\ 2 Department of Biology, Xinzhou Teachers University, Xinzhou, Shanxi, P. R. China \\ Corresponding Author: Jiufeng Wei \\ Email address: wjfeng@nwsuaf.edu.cn
}

According to current molecular evidence, the Chionaspis pinifoliae heterophyllae species complex has been recognized as 10 cryptic species. In this study, based on climatic variables, we construct potential distribution maps for seven cryptic species. This was done to assess the main environmental factors that have contributed to the distribution map and test the degree of niche overlap across the seven cryptic species. We used software MaxEnt to build the climatic niche models based on climatic variables. For these models, we estimated the similarities and differences of the niches across the cryptic species. By comparing the potential distribution model of each cryptic species, our results suggested parapatric, sympatric and allopatry populations for this cryptic species complex. Our results showed high variability in niche overlap, and more often niche conservatism than niche divergence. The results support the current species delimitation of the Chionaspis pinifoliae heterophyllae complex by molecular information. Our study supports the hypothesis that the niche overlap in the sympatric population is higher than that of the allopatry population. Moreover, the results provide baseline data and a distribution range to facilitate the further control of these insects and formulate quarantine measures. 
1 Climatic niche comparison across a cryptic species complex

2 Qing Zhao ${ }^{1 *}$, Hufang Zhang ${ }^{2 *}$, Jiufeng Wei ${ }^{1}$

3 1. Department of Entomology, Shanxi Agricultural University, Taigu, Shanxi, P.R. China

4 2. Department of Biology, Xinzhou Teachers University, Xinzhou, Shanxi, P.R. China

$5 *$ These authors contributed equally to this work.

6 Corresponding Author: Jiufeng Wei

7 Email address: corresponding author,wjfeng@nwsuaf.edu.cn

8

9 ABSTRACT

\section{Introduction}

According to current molecular evidence, the Chionaspis pinifoliae heterophyllae species complex has been recognized as 10 cryptic species. In this study, we construct potential distribution maps for seven cryptic species based on climatic variables. This was done to assess the main environmental factors that have contributed to the distribution map and test the degree of niche overlap across the seven cryptic species. We used MaxEnt to build the climatic niche models under climatic variables. For these models, the similarities and differences of the niches across the cryptic species were estimated. By comparing the potential distribution model of each cryptic species, our results suggested parapatric, sympatric and allopatry populations for this cryptic species complex. Our results showed high variability in niche overlap, and more often niche conservatism than niche divergence. The current species delimitation of the Chionaspis pinifoliae heterophyllae complex by molecular information and the hypothesis that the niche overlap in the sympatric population is higher than that of the allopatry population were supported based on the findings. This study will provide baseline data and a distribution range to facilitate the further control of these insects and formulate quarantine measures.

Ecological factors play an important role in speciation events by providing sources of selection that drive micro-evolutionary change and by imposing constraints that limit organism performance (Barrett \& Hoekstra,2011; Pyron \& Burbrink, 2009; Rissler \& Apodaca, 2007). If evolutionary lineages become geographically isolated during periods of climatic change, ecological components can also directly drive speciation. Nevertheless, species can also maintain their ancestral niche, which is referred to as niche conservatism (Wiens, 2004; Peterson et al., 1999; Peterson, 2011). The study of how species fluctuate in their requirements for and tolerance 
of such factors has advanced, in part, due to the continued theoretical development and quantification of the ecological niche of species (Soberón, 2007). Many methods have been proposed to test niche conservatism (Wiens, 2004) and defined the climatic variables that constrain the distribution of species. Ecological niche modeling (ENM) is a relatively new method that has been used as a powerful tool to examine niche divergence and conservatism (Culumber \& Tobler, 2016; Scriven et al., 2016). Spatially unequivocal environmental data and models allow for extensive scale tests about whether speciation is associated with niche divergence or whether closely related species tend towards niche conservatism (McCormack et al., 2010). The development of ENM has promoted the extraction of ecological niche characteristics, which can help identity the niche limits of cryptic species (Wellenreuther et al., 2012). Niche features have been applied to improve inferences about species boundaries and speciation mechanisms. Recently, a principal component analysis (PCA) method was present by Broennimann et al (2012) that transforms the investigated environmental variables into a twodimensional space identited by the first and second principal components. The niche overlaps value was measured by this approach that provides a relatively reliable way to test the niche divergence and niche conservatism hypotheses (Ahmadzadeh et al., 2013).

Cryptic species are a distinct but morphologically similar species that were classified as being a single one (Pfenninger \& Schwenk, 2007). There species are not only important for taxonomic reasons, but also hold significant meaning in terms of biogeography (Pfenninger \& Schwenk, 2007) and biodiversity (Oliver et al., 2009). The armored scale insects (Hemiptera, Diaspididae) are a family of over 2,650 described species according to current records (García et al., 2016). The Chionaspis pinifoliae heterophyllae species complex has only included two species, which are C. heterophyllae Cooley and C. pinifoliae (Fitch), since 1921 (MacGillivray, 1921). However, current reanalysis of species diversity within this group by Gwiazdowski and his colleagues (Gwiazdowski et al., 2011) that suggested the presence of at least 10 closely related species, which have been delimited as cryptic species. These species are origin to North America (Vea et al., 2012) and are considered pests on Pinus in forests and ornamental settings (Miller \& Davidson, 2005). The adaptive deme formation (ADF) hypothesis proposes that a population may adapt to an individual host resulting in "a series of semi-isolated subpopulations, or demes" (Edmunds \& Alstad, 1978). Many studies support ADF leading to multiple closely related cryptic species, such as armored scales (Anderson et al., 2010; Cook \& Rowell, 2007). The result of research of Gwiazdowski et al. (2011) also supports this hypothesis. However, this 
study did not consider the ecological or climatic requirements of the species. Identifying the ecological niches of cryptic species is important to understanding the creation and maintenance of biodiversity (Oliver et al., 2009).

In the current study, ENMs and ordination techniques were used to characterize the ecological niches of the members of the Chionaspis pinifoliae heterophyllae species complex and test similarities between them. The main questions addressed in this study were: (1) What are the main environmental variables that constrain the potential distributions of members of $C$. pinifoliae heterophyllae? (2) Do cryptic species share the same ecological conditions? (3) What is the potential mechanism of geographic speciation of the allopatric and sympatric populations? The closely related species will result in high levels of similarity, ecological niche and spatial overlap. In addition, with niche divergence as a speciation mechanism, we would expect the ecological niches of closely related species to differ significantly. Because the cryptic species have similar morphological and physiological features, their ecological niches would be similar. However, because of the different adaptations to different environmental conditions, the nonequivalence of their ecological niches would be expected in the current study.

\section{Methods}

\section{Study area and species}

The Chionaspis pinifoliae-Chionaspis heterophyllae $(\mathrm{CPCH})$ species complex inhabits across the USA, Mexico, and parts of Canada. Distribution data for $\mathrm{CPCH}$ were taken from Gwiazdowski et al. (2011). S3, S4, and S9 could not be included because of the low number of available sample locations $(<5)$. Duplicate occurrences with the same geographic coordinates were removed using ENMtools software (Warren et al., 2010). Occurrence records are often biased towards areas that are easily accessible or are near cities or other areas of high population density, thus, to remove spatial autocorrelation and the sampling bias, a grid of $5 \times 5 \mathrm{~km}$ cells was created. A single point from each cell containing one or more sampling points was randomly selected. After filtering, 201 localities were retained in the final analysis, including S1 (29 points), S2 (21 points), S5 (8 points), S6 (22 points), S7 (22 points), S8 (13 points) and S10 (160 points). The distribution of the localities used in the study is presented in Figure 1.

\section{Climate variables}

Climate data for 19 environmental variables were obtained from the WorldClim database (Hijmans et al., 2005; http://www.worldclim.org). Climate data represent the biologically 
96

relevant summaries of means and variation in precipitation and temperature recorded between 1950 and 2000. All climatic variables had a spatial resolution of 30 arc seconds (approximately 1 $\mathrm{km}$ resolution at the equator). Since strong covariance among environmental variables can cause "overfitting" of the model and affect model accuracy in ENM (Synes \& Bsborne, 2011; Boria et al., 2014), it is important to minimize such correlations using dimension-reduction techniques. Thus, a Pearson's correlation test based on all 19 climate variables was conducted for all species' presence points, andused to exclude highly correlated variables $(|r|>0.80)$ from our models (Table S1). Six predictor variables were used in subsequent processing, which were Mean Diurnal Range (Bio2), Mean Temperature of the Wettest Quarter (Bio8), Mean Temperature of the Driest Quarter (Bio9), Annual Precipitation (Bio12), the Precipitation of the Warmest Quarter (Bio18) and the Precipitation of the Coldest Quarter (Bio19).

\section{Ecological niche modeling (ENM)}

Ecological niche modeling uses environmental variables and occurrence data to simulate suitable environmental conditions for focus species. Researchers have proposed the use of many different software packages to study invasion biology (Waltari \& Hickerson, 2012; Guo et al., 2013), conservation biology (Préau et al., 2018), biodiversity research (Thuiller et al., 2006) and ecological niches (Chetan et al., 2014; Reeves \& Richards, 2011). Of the species distribution model algorithm methods, Maxent (Maximum Entropy) has proved powerful when modeling rare data with narrow ranges and only with presence-only data (Elith et al., 2011; Qin et al., 2017). The MaxEnt version (version 3.3.3k) (Phillips et al., 2006) was used to construct SDMs for all species.

The default MaxEnt settings were suggested to produce overfitted models in a recent study (Radosavijevic \& Anderson, 2014). Hence, in order to balance the model fit and predictive ability, the R package ENMeval was used to select the optimal combination of two important MaxEnt's parameters which are the value of the regularization multiplier and the combination of feature classes (Warren \& Seifert, 2011; Muscarella et al., 2014). The "checkerboard2" approach was employed to calculate the standardized Akaike information criterion coefficient (AICc), and models with the lowest delta AICc score were selected to run the final MaxEnt models. Five different feature class combinations (1) L; (2) LQ; (3) LQH; (4) LQHP, (5) LOHPT (where L = linear; $\mathrm{Q}=$ quadratic; $\mathrm{H}=$ hinge; $\mathrm{P}=$ product and $\mathrm{T}=$ threshold) were tested, meanwhile, the regularization multiplier was varied from 0.5 to 4 in increments of 0.5 . The result are shown in Table S2 and Figure S1. The logistic output was used in the model, which was a continuous map 
128

129

130

131

132

133

134

135

136

137

138

139

140

141

142

143

with an estimated probability of presence between 0 (lowest) to 1 (highest). The percentages contribution and permutation importance of the environmental variables were calculated, and jackknife procedures were executed to evaluate the relative importance variable in MaxEnt. The model was run with a convergence threshold of $10^{-5}$, maximum iterations of 5,000 and maximum number of background points of 10,000. The MaxEnt model was created based on the 10-fold replicates with cross-validation method. The remaining model values were set to default values (Penado et al., 2016).

The area under the receiver operating characteristic (ROC) curve (AUC) is a widely used statistic for the measurement of MaxEnt performance (Merow, Smith \& Silander, 2013; Wei et al., 2018). However, this method has been criticized for its equal weighting of omission and commission errors (Lobo et al., 2008; Peterson et al., 2008). In addition, this method can not provide information about the spatial distribution of model errors the total extent to which models are carried out highly influences the rate of well-predicted absences, and the AUC scores (Lobo et al., 2008). Thus, an alternative Partial ROC metric approach was employed to model evaluations (Peterson et al., 2008). Partial ROC statistics were implemented using online the Niche Toolbox site (http://shiny.conabio.gob.mx:3838/nichetoolb2/) with 1000 replicates and E $=0.05$. To improve predictions made in this study, the predicted continuous suitability maps by MaxEnt were converted into suitable/unsuitable areas (binary habitat) using an applied threshold. Here, the lowest presence threshold (LPT $=$ minimum training presence threshold of MaxENT) was selected for each species (Pearson et al., 2007; Wisz et al., 2008). This threshold can identify the minimum predicted area possible whilst maintaining zero omission error in the training dataset.

\section{Calculating niche overlap in E-space}

An assessment of niche overlap was used to quantify the niches shared within the species complex was done through. Climate niche overlaps among species were estimated using the PCA-env method proposed by Broennimann et al. (2012). Principal component analysis (PCA) was used to transform the environmental space of the investigated or selected environmental variables into a two-dimensional space defined by the first and second principal components (Strubbe et al., 2015). The two-dimensional environmental space was then projected onto a $100 \times 100$ PCA grid of cells bounded by the minimum and maximum PCA values in the background data. This method also corrects the potential sampling bias in occurrence records using a smooth kernel density function (Broennimann et al., 2012). The niche overlap within the 
160

161

162

163

164

165

166

167

168

169

170

171

172

173

174

175

176

177

178

179

180

181

182

183

184

185

186

187

188

189

190

191

species complex was measured by the mean of Schoener's D directly from the ecological niche space (Warren et al., 2008). The Schoener's D is an index which varies from 0 (no overlap) to 1 (overlap). In addition, there are two common statistical tests considering hypotheses (niche equivalence test and niche similarity test) of niche divergence or conservatism.

A niche equivalence test was applied to estimate whether the ecological niches of the pairs of the species complex are significantly different from each other and whether the two niche spaces are interchangeable. The niche equivalence was determined by comparing the niche overlap values (D) of pairs of a species complex to a null distribution of 100 overlap values. If the niche overlap value fell outside the $95 \%$ of the null hypotheses, the equivalency of the two niches could be rejected. Niche similarity test compares the niche overlap of one range randomly distributed over its background, while keeping the other unchanged $(1 \rightarrow 2)$, and then carries out the reciprocal comparison $(1 \leftarrow 2)$. For the similarity test, a $p$ value $>0.05$ was considered to indicate that niches were no more similar than expected by chance. Niche similarity tests wre used in the current study to estimate niche differentiation. The overlap value between two ENMs was either above the $95 \%$ confidence interval of the null hypothesis, supporting niche conservatism, or below the $95 \%$ confidence interval of the null hypothesis, supporting niche divergence.

In addition, the null hypothesis of niche equivalency was also tested using ENMTools 1.4.4 (Warren et al., 2010). ENMTools uses MaxEnt to generate an ENM from each species, and then uses the model and predicted suitability scores generated by Maxent for each species to calculate niche equivalency test. This test is based on the metrics of niche overlap (Schoener's $D$ and $I$ ) that ranging vary from 0 (no overlap) to 1 (complete overlap) (Warren et al., 2008). The ecological interpretation of Schoener's $D$ assumes that the suitability scores are proportional to species abundance, whereas Schoener's $I$ treats the two ENMs as probability (Warren et al., 2010). The null hypothesis that the two species pairs have similar niches is accepted if the niche overlap of both species pairs is outside of the $95 \%$ confidence interval. When niche overlap between two groups does not fall within the $95 \%$ confidence interval and Schoener's $D$ and $I$ are less than our assumption value, then the groups are separated (Warren et al., 2008).

All GIS analyses were performed using ArcGIS version 10.2.1 (ESRI). All statistical analyses were performed in R using scripts from Broennimann et al. (2012), now available in the 'ecospat' R package (Di Cola et al., 2017).

Results

PeerJ reviewing PDF | (2018:07:29870:5:0:NEW 23 Apr 2019) 
192

193

194

195

196

197

198

199

200

201

202

203

204

205

206

207

208

209

210

211

212

213

214

215

216

217

218

219

220

221

222

223

\section{Niche modeling and responses to climate variables}

The partial ROC tests indicated a significant predictive ability for the models for all species $(\mathrm{P}<0.001)$ (Figure S2). The percentages of the variable contribution by members of the Chionaspis pinifoliae-Chionaspis heterophyllae species complex to the model construction are shown in Table 1. To visualize the suitable habitat areas of the species complex, model predictions were imported into a geographic information system and the areas were reclassified into two arbitrary categories of habitat suitability: unsuitable habitat (<threshold) and habitat suitability areas (threshold-1). The threshold is shown in Table 1. The distribution of the cryptic species is determined by different responses to the environment (Figure 2 and Figure 3 ).

Suitable habitat areas for S1 were found at the high mean temperature of the driest quarter (Bio9) $\left(-1-21^{\circ} \mathrm{C}\right)$ and precipitation of the coldest quarter (Bio19) (200-5000 mm). Both climate variables are the most important predictors for their potential distribution (Figure 2 and Table 2). The mean temperature of the driest quarter (Bio9) was also an important environmental factor constraining the distribution of $\mathrm{S} 2$, with the highest suitability at values between $14.5^{\circ} \mathrm{Cand}$ $21^{\circ} \mathrm{C}$. The distribution of S5 was mainly constrained by the mean diurnal range (Bio2) (14.5$20.5^{\circ} \mathrm{C}$ ). S6 showed optimum suitability at the mean temperature of the wettest quarter (Bio8) ($5-5^{\circ} \mathrm{C}$ ) and at the mean temperature of the driest quarter (Bio9) $\left(-3-31^{\circ} \mathrm{C}\right)$. S7 was mainly constrained by the precipitation of the coldest quarter (Bio19) (100-900 mm). The mean temperature of wettest quarter (Bio8) was an important climatic factor constraining the distribution of $\mathrm{S} 8$, with the highest suitability at values between $-2^{\circ} \mathrm{C}$ and $12^{\circ} \mathrm{C}$. Suitable habitat areas for $\mathrm{S} 10$ were found for the mean diurnal range $(\mathrm{Bio} 2)$ of $2{ }^{\circ} \mathrm{C}-15^{\circ} \mathrm{C}$, and the mean temperature of the wettest quarter $(\mathrm{Bio} 8)$ of $-20^{\circ} \mathrm{C}-30^{\circ} \mathrm{C}$.

Relative to other environmental variables, temperature factors including mean diurnal range (Bio2) and mean temperature of the wettest quarter (Bio8) were the main factors affecting the distribution of this species complex.

\section{Niche overlap, niche equivalency and similarity}

The results from the niche overlap suggest high variations exist in the environmental space inhabited by the different $\mathrm{CPCH}$ (Table 3). The PCA-env of niche similarity (Figure S3-S6) in the species pairs of two PCA axes explaining from 58.7\% (S1 vs S5) to 77.48\% (S6 vs S10) of the total variation of the seven climatic variables. A great overlap was shown for certain pairs of species, such as: S6 and S8 (0.649), S2 and S5 (0.488), S2 and S7 (0.477) and S7 and S8 (0.477). However, the niche overlap of the other species pairs was relatively low, such as S1 and S6 
224 (0.014), S1 and S7 (0.042), S2 and S8 (0.0146), and S2 and S6 (0.097). The low niche overlap 225 suggested that they occupy considerably different environmental niches. All niche overlap values 226 are illustrated in Table 3.

227 According to the identify test, the true calculated niche overlap of all species pairs, are 228 outside of the 95\% confidence interval of the null hypothesis (Figure S7) and confirm the 229 separation between them. Thus, these species pairs showed that the species' ENMs were not 230 equivalent.

231 The above test results indicate that the niches of $\mathrm{CPCH}$ species complex are similar, but 232 they are not identical.

\section{Geography of speciation}

The degree of geographic overlap by ENM between the species pairs provides evidence that suggests that some sympatric, parapatric and allopatric species exist in our analysis based on the similarity test. Of the 21 total pairs, two cases of parapatry between species pairs in these species complex were known (S1 and S7; and S1 and S10). Four cases of allopatry between species pairs in these species complex were known (S1 and S2; S1 and S5; S1 and S6; and S1 and S8). In addition, 15 species pairs in the species complex were found to exhibit sympatry. The parapatric species pairs had a small niche overlap (S1 vs $\mathrm{S} 7=0.041$; S1 vs $\mathrm{S} 10=0.231$ ). As predicted, all allopatric (except S1 vs S5 = 0.476) species pairs also suggest a small niche overlap. All speciation types also showed niche divergence in same direction.

243

\section{Discussion}

In this study, the environmental constraints for the current distribution of the members of the $\mathrm{CPCH}$ species complex were identified by applying ecological niche modeling and ordination techniques. The distribution range of the $\mathrm{CPCH}$ species complex in the North American continent mirrors the various environmental conditions to which they are adapted to. Additionally, they may also reflect the physiological differences between them. Temperature factors were the most significant limiting factor in the distribution of all $\mathrm{CPCH}$. Higher temperatures could increase the fitness and abundance of the scale insect (Dale \& Frank, 2017), thereby possibly increasing their chances of survival in extreme conditions.

Our results have shown that the distribution of each member of the $\mathrm{CPCH}$ complex is constrained by a set of environmental conditions. Climate change will directly affect the potential distribution of species, the ranges of which are strongly constrained by temperature and precipitation (Chen et al., 2011). The potential distribution of the members of the $\mathrm{CPCH}$ 
256

257

258

259

260

261

262

263

264

265

266

267

268

269

270

271

272

273

274

275

276

277

278

279

280

281

282

283

284

285

286

287

complex under climate change is not only limited by climate variables but is also affected by other factors, such as species dispersal mechanisms, host-plant availability and human-mediated transport. The results provide a distribution range to facilitate the further control of these insects and formulate quarantine measures when an invasion by a member of this complex occurs.

Given the wide variation in the environmental conditions at locations where the members of the $\mathrm{CPCH}$ complex can be found, a low niche overlap between some members of this complex was expected. The low values of niche overlap between S1 and S7, S1 and S6, S2 and S6, and between S2 and S8, were also reflected in their different climate variable constraints. The result of the niche equivalency test between all pairs suggested a lack of ecological exchangeability. This result is in agreement with a previous study (Scriven et al., 2016) that morphological similarity does not necessarily equate to ecological equivalence. The result of the niche similarity test shows that the $\mathrm{CPCH}$ complex shares more climate niche characteristics than would be expected from random occurrence. In summary, the results show that there is a close relationship between these species, and they are likely to share climatic niche spaces. It is further confirmed that the members of the $\mathrm{CPCH}$ complex are closely related but are of different taxa. The differences in the environmental constraints of the different $\mathrm{CPCH}$ complex are also reflected in the niche similarity, overlap and equivalency results. In our analysis, the significant differences in the niche spaces reflect the reported taxonomic divisions within the $\mathrm{CPCH}$ complex (Gwiazdowski et al., 2011). Previous research has used differences in niches to support species delimitation (Aguirre-Gutierrez et al., 2013; Raxworthy et al., 2007). Our results support the current species delimitation of the CPCH complex by molecular methods (Gwiazdowski et al., 2011; Vea et al., 2012). These cryptic species are morphologically similar, but the climatic ecological characteristics have caused differentiation. Our results are consistent with other research that niches are similar but not equivalent across closely related species (AguirreGutierre et al., 2013).

There are three traditional geographic categories for the phenomenon of speciation, which are allopatric (non-overlapping), sympatric (overlapping) and parapatric (adjoining) speciation, which depend on the degree of range overlap between species pairs during the speciation process (Zheng et al., 2017; Bultin et al., 2008). The speciation of different geographic categories was identified by the potential distribution modelling in recent studies. Our study supports the hypothesis that the niche overlap in the sympatric population was greater than that of the allotropic population (Hochkirch \& Groning, 2012). 
288

289

290

291

292

293

294

295

296

297

298

299

300

301

302

303

304

305

306

307

308

309

310

311

312

313

314

315

316

317

318

319

Considerable overlap was found in the climatic niches of the different members of the $\mathrm{CPCH}$ complex (Table 3), such as S6 vs S8, S1 vs S5 and so on. These findings suggest that the divergent natural selection along climatic axes of the niche had played a limited role in the development of some pairs of this species complex. Many studies on a wide variety of taxa have suggested that ecological divergence plays an important role in sympatric populations (Via, 2001; Schliewen et al., 2001). Our results in the current paper suggest the lack of a large proportion of niche divergence between species pairs. These results are in accordance with the expectation that, given the climatic conditions available to them, closely related pairs will not be equivalent in their climatic niches, but will typically be more similar than expected. Our results agree with previous studies on the niche conservatism of sister species or species complexes (Graham et al., 2004; Silva et al., 2014). In our study, sympatric species pairs had very similar environmental niches or relatively high niche overlap, e.g., S6 vs S8 (Table 3, Figure 2). This result suggests that these cryptic species likely experienced similar environmental pressures throughout their evolutionary history. Furthermore, the climatic niches are less likely to be influenced by competition and may thus represent interspecific differences in the fundamental niche. However, the larger niche overlap must lead to competition in sympatric and parapatric species pairs. Direct competition between these cryptic species is unlikely to exist (e.g., feeding), but there is potential competition for resources (e.g., host plants). The differences found in their use of forage plants may possibly be driven by competition and could reflect differences in their realized niches, rather than their fundamental niches.

Allopatric speciation is essentially a spatial process where two populations become genetically isolated due to geography, and thus the species pairs would have a relatively small niche overlap. The results of the current study suggest that some allopatric species pairs had a relatively small niche overlap, such as: S1 vs S6 and S1 and S8 (Table 3, Figure 2). These results indicate that their niches belong to different environmental conditions. A hypothesis has been proposed that allopatric species may originate when a geographic barrier (i.e., an area of unsuitable environmental conditions between two sets of populations) develops faster than adaptation to these new ecological conditions. S1 is mainly distributed in southeastern North America and S6 located in western North America. There is a relatively large distance between the two species, with significant geographical isolation. Similarly, there is a larger geographic barrier between S1 and S8. In addition, the host plants of S1 are Pinus sylvestris, P. nigra, P. palustris, P. echinata, P. pungens, P. taeda, P. virginana, P. elliottiii, and P. rigida, while the 
320 host plants of S6 are $P$. torreyana, $P$. strobiformis, $P$. lambertiana, $P$. ponderosa, $P$. contorta, $P$. 321 quadrifolia, P. attenuate, P. halepensis, P. jeffreyi, P. sabineana, and P. torreyana var. insularis. 322 The host plants of S8 are Pinus undet, P. radiate, P. lambertiana, P. attenuate, P. ponderosa, $P$. 323 contorta and Pseudotsuga menziesii. The species pairs have completely different host plants. 324 Therefore, there may be a strict dietary isolation between the two species pairs. The distribution 325 of host plants might determine the distribution of insects in our studies (Provencher et al., 2005). 326 However, since populations on each side of the barrier would still have the same niche in cryptic 327 species, it would have been niche conservatism that maintained the allopatric distribution. In our 328 study, a small but clear overlap between the suitable areas defined by the ENMs of the allopatric 329 sister taxa (Kozak \& Wiens et al., 2006) would have been found. Our study also supports the 330 hypothesis that niche conservatism may be generally important in allopatric speciation because it 331 will limit adaptation to ecological conditions at the geographic barrier (Warren et al., 2008). 332 Parapatric species pairs (e.g., S2 vs S8, S5 vs S6, S2 vs S6) also show a relatively low niche 333 overlap value. The underlying mechanism is similar to that of allopatric species distribution.

334

335

336

337

338

339

340

341

342

343

344

345

346

347

348

349 350

\section{Conclusion}

In summary, our results suggest that niche conservatism is common in this cryptic species complex and that these species occupy different climatic niches. The results suggest that allopatric species pairs had a relatively small niche overlap, which indicates that their niches are adapted to different environmental conditions. In addition, the results in our current paper suggest that there is a lack of a large proportion of niche divergence between sympatric species pairs. The results suggest that these cryptic species had likely experienced similar environmental pressures throughout their evolutionary history. In current study, the niche comparisons were implemented only using the Broennimann et al. method (2012). However, other approaches, such as ENMtools (Warren et al., 2010) or using more sophisticated methods of multivariate analysis, such as measures of the multidimensional overlap in species' niche positions and breadths (Blonder et al., 2014) might provide much clearer results. Additional, only climate condition was consider in current work, however, other niche factor such as dispersal ability (Guisan \& Thuiller, 2005), Interspecific interactions (Gao \& Reitz, 2017) and Host-plant availability (Ning, 2017), also affected the precision of the model. Thus, these factors should be consideration in future research. 
352

353

354

355

356

357

358

359

360

361

362

363

364

365

366

367

368

369

370

\section{References}

Anderson JC, Normark BB, Morse GE, Gruwell ME. 2010. Cryptic diversity in the Aspidiotus nerii complex in Australia. Annals of the entomological society of America 103, 844-854.

Aguirre-Gutiérrez J, Serna-Chavez HM, Villalobos-Arambula AR, Rosa JAP, Raes N. 2013. Similar but not equivalent: ecological niche comparison across closely-related Mexican white pines. Diversity and Distribution 21, 245-257.

Ahmadzadeh F, Flecks M, Carretero MA, Bohme W, Ilgaz C, Engler JO, Harris DJ, Uzum N, Rodder D. 2013. Rapid Lizard radiation lacking niche conservatism: Phylogeny and ecological diversification within a complex landscape. Journal of Biogeography 40(9), 1807-1818.

Barrett RDH, Hoekstra HE. 2011. Molecular spandrels: tests of adaptation at the genetic level. Nature Reviews Genetics 12(11), 767-780.

Blonder B, Lamanna C, Violle C, Enquist BJ. 2014. The n-dimensional hypervolume. Global Ecology and Biogeography 23, 595-609.

Broennimann, Fitzpatrick MC, Pearman PB, Petitpiette B, Pellissier L, Yoccoz NG, Thuiller W, Fortin MJ, Randin C, Zimmermann NE, Graham CH, Guisan A. 2012. Measuring ecological niche overlap from occurrence and spatial environmental data. Global ecology and biogeography 21(4),481-497.

Di Cola V, Broennimann O, Petitpierre B, Breiner FT, D’Amen M, Randin C, Engler R, Pottier J, Pio D, Dubuis A, Mateo RG, Hordijk W, Salamin N, Guisan A. 2017. Ecospat: an R package to support spatial analyses and modeling of species niches and distributions. Ecography 40, 001-014.

Bultin KR, Galindo J, Grahame JW. 2008. Sympatric, parapatric or allopatric: the most important way to classify speciation? Philosophical transactions of the royal society of London series b-biological sciences 363, 2997-3007.

Boria RA, Olson LE, Goodman SM, Anderson RP. 2014. Spatial filtering to reduce sampling bias can improve the performance of ecological niche models. Ecological Modelling 275, 73-77.

Chetan N, Praveen KK, Vasudeva GK. 2014. Delineating ecological boundaries of hanuman langur species complex in peninsular India using MaxEnt modeling approach. PLoS ONE 9(2), e87804.

Chen IC, Hill JK, Ohlemuller R, Roy DB, Thomas CD. 2011. Rapid range shift of species associated with high levels of climate warming. Science 333(6045), 1024-1026).

Cook LG, Rowell DM. 2007. Genetic diversity, hostspecificity and unusual phylogeography of a cryptic, host associated species complex of gall-inducing scale insects. Ecological Entomology 32, 506-515.

Culumber ZW, Tobler M. 2016. Ecological divergence and conservatism: spatiotemporal patterns of niche 
evolution in a genus of livebearing fishes (Poeciliidae: Xiphophorus). BMC evolutionary Biology 16(44), $1-16$.

Dale AG, Frank SD. 2017. Warming and drought combine to increase pest insect fitness on urban trees. PLoS ONE 12(3), e0173844.

Di Cola V, Broennimann O, Petitpierre B, Breiner FT, D'amen M, Randin C, Engler R, Pottier J, Pio D, Dubuis A. 2017. ecospat: an $R$ package to support spatial analyses and modeling of species niches and distributions. Ecography 40, 774-787.

Edmunds GF, Alstad DN. 1978. Coevolution in insect herbivores and conifers. Science 199, 941-945.

Elith J, Phillips SJ, Hastie T, Dudik M, Chee YE, Yates CJ. 2011. A statistical explanation of MaxEnt for ecologists. Diversity and Distribution 17, 43-57.

García M, Morales M, Denno BD, Miller DR, Miller GL, Ben-dov Y, Hardy NB. 2016. ScaleNet: A Literaturebased model of scale insect biology and systematics. http://scalenet.info [accessed: 2018]

Gao YL, Reitz SR. 2017. Emerging themes in our understanding of species displacements. Annual review of entomology 62, 165-183.

Guisan A, Thuiller W. 2005. Predicting species distribution: offering more than simple habitat model. Ecology letters 8(9), 993-1009.

Graham CH, Ron SR, Santos JC, Schneider CJ, Moritz C. 2004. Integrating phylogenetics and environmental niche models to explore speciation mechanisms in dendrobatid frogs. Evolution 58, 17811793.

Guo WY, Lambertini C, Li XZ, Meyerson LA, Brix H. 2013. Invasion of old world Phragmites australis in the new world: precipitation and temperature patterns combined with human influences redesign the invasive niche. Global Change Biology 19(11), 3406-3422.

Gwiazdowski RA, Vea MI, Andersen CJ, Normark BB. 2011. Discovery of cryptic species among North American pine-feeding Chionaspis scale insects (Hemiptera: Diaspididae). Biological Journal of the Linnean Society of London 104, 47-62.

Hijmans RJ, Cameron SE, Parra JL, Jones PG, Jarvis A. 2005. Very highly resolution interpolated climate surfaces for global land areas. International journal of climatology 25, 1965-1978.

Hochkirch A, Gröning J. 2012. Niche overlap in allotropic and syntopic populations of sexually interacting ground-hopper species. Insect Science 19, 391-406.

Kozak KH, Wiens JJ. 2006. Does niche conservatism drive speciation?A case study in North American salamanders. Evolution 60, 2604-2621.

Lobo JM, Jiménez-Valverde A, Real R. 2008. AUC: a misleading measure of the performance of predictive 
distribution models. Global Ecology and Biogeography 17, 145-151.

MacGillivray A D, 1921. The Coccidae. Tables for the Identifcation of the Subfamilies and Some of the More Important Genera and Species, together with Discussions of their Anatomy and Life History. Scarab, Urbana, IL, $520 \mathrm{pp}$.

McCormack JE, Zellmer AJ, Knowles LL. 2010. Do niche divergence accompany allopatric divergence in Aphelocoma jays as predicted under ecological speciation? Insights from tests with niche models. Evolution 64, 1231-1244.

Merow C, Smith MJ, Silander JA. 2013. A practical guide to MaxEnt for modeling species'distributions: what it does, and why inputs and settings matter. Ecography 36 (10), 1058-1069.

Miller DR, Davidson JA. 2005. Armored Scale Insect Pests of Trees and Shrubs (Hemiptera: Diaspididae). Cornell University Press, Ithaca, NY, 456 pp.

Mwase ET, Stensgaard AS, Nsakashalo-Senkwe M, Mubila L, Mwansa J. 2014. Mapping the geographical distribution of lymphatic filariasis in Zanbia. PLoS Neglectted Tropical Diesases 8(2), e2714.

Muscarella R, Galante PJ, Soley-Guardia M, Boria RA, Kass JM, Uriarte M, Anderson RP. 2014. ENMeval: an R package for conducting spatially independent evaluationsand estimating optimal model complexity for MaxEnt ecological niche models. Methods in Ecology and Evolution 5(11),1198-1205

Ning SY, Wei JF, Feng JN. 2017. Predicting the current potential and future worldwide distribution of the onion maggot, Delia antique using maximum entropy ecological niche modeling. PLOS ONE 12(2), e0171190.

Oliver PM, Adams M, Lee MSY, Hutchinson MN, Doughty P. 2009. Cryptic diversity in vertebrates: molecular data double estimates of species diversity in a radiation of Australian lizards (Diplodactylus, Gekkota). Proceedings of the Royal Society B-Biological Sciences 276, 2001-2007.

Pearson RG, Raxworthy CJ, Nakamura M, Peterson AT. 2007. Predicting species distributions from small numbers of occurrence records: a test case using cryptic geckos in Madagascar. Journal of Biogeography 34(1), 102-117.

Peterson AT, Soberón J, Sanchez-Cordero V. 1999. Conservatism of ecological niches in evolutionary time. Science 285, 1265-1267.

Peterson AT, Papes M, Soberón J. 2008. Rethinking receiver operating characteristic analysis applications in ecological niche modeling. Ecological modeling 213, 63-72.

Peterson AT. 2011. Ecological niche conservatism: a time-structured review of evidence. Journal of Biogeography 38, 817-827.

Penado A, Rebelo H, Goulson D. 2016. Spatial distribution modeling reveals climatically suitable areas for 
bumblebees in undersampled parts of the lberian Peninsula. Insect Conservation \& Diversity 5, 391-401.

Pfenninger M, Schwenk K. 2007. Cryptic animal species are homogeneously distributed among taxa and biogeographical regions. BMC Evolution and Biology 7, 1-6.

Phillips SJ, Anderson RP, Schapire RE. 2006. Maximum entropy modeling of species geographic distributions. Ecological Modelling 190, 231-259.

Préau C, Trochet A, Bertrand R, Isselin-Nondereu F. 2018. Modeling potential distributions of three European amphibian species comparing ENFA and Maxent. Herpetological Conservation and biology 13(1), 91-104.

Provencher LM, Morse GE, Weeks AR, Normark BB. 2005. Parthenogenesis in the Aspidiotus nerii complex (Hemiptera: Diaspididae): a single origin of a worldwide, polyphagous lineage associated with Cardinium bacteria. Annals of the Entomological Society of America 98, 629-635.

Pyron RA, Burbrink FT. 2009. Lineage diversification in a widespread specie: roles for niche divergence and conservatism in the common kingsnake, Lampropeltis getula. Molecular Ecology 18, 3443-3457.

Qin L, Liu B, Guo QS, Bussmann RW, Ma FQ, Jian ZJ, Xu G, Pei SX. 2017. Maxent modeling for predicting impacts of climate change on the potential distribution of Thuja sutchuenensis Franch., an extremely endangered conifer from southwestern China. Global ecology and conservation 10, 139-146.

Radosavljevic A, Anderson RP. 2014. Making better Maxent models of species distributions: complexity, overfitting and evaluation. Journal of Biogeography 41(4), 629-643.

Raxworthy CJ, Ingram CM, Rabibisoa N, Pearson RG. 2007. Applications of ecological niche modeling for species delimitation: a review and empirical evaluation using day geckos (Phelsuma) from Madagascar. Systematic Biology 56(6), 907-923.

Reeves PA, Richards CM. 2011. Species delimitation under the general lineage concept: an empirical example using wild north American hops (Cannabaceae: Humulus llupulus). Systematic Biology 60, 45-59.

Rissler LJ, Apodaca JJ. 2007. Adding more ecology into species delimitation: ecological niche models and phylogeography help define cryptic species in the black salamander (Aneides flavipunctatus). Systematic Biology 56(6), 924-942.

Runquist RDB, Lake T, Moeller DA. 2019. Species distribution models throughout the invasion history of Palmer amaranth predict region at risk of future invasion and reveal challenges with modeling rapidly shifting geographic ranges. Scientific Reporsts, 9, 2426.

Soberón J. 2007. Grinnellian and Eltonian niches and geographic distributions of species. Ecology Letter 10, $1115-1123$.

Scriven JJ, Whitehorn PR, Goulson D, Tinsley MC. 2016. Niche partitioning in a sympatric cryptic species 
complex. Ecology and Evolution 6(5), 1328-1339.

Schliewen UK, Rassman K, Markmann J, Tautz D. 2001. Genetic and ecological divergence of a monophyletic cichlid species pair under fully sympatric conditions in Lake Ejagham, Cameroon. Molecular Ecology 10, 1471-1488.

Silva DP, Vilela B, De Marco PJ, Nemesio A. 2014. Using Ecological Niche Models and Niche Analyses to Understand Speciation Patterns: The Case of Sister Neotropical Orchid Bees. PLoS ONE 9(11), e113246.

Synes NW, Bsborne PE. 2011. Choice of predictor variables as a source of uncertainty in continental-scale species distribution modelling under climate change. Global Ecology and Biogeography 20(6), 904-914.

Strubbe D, Beauchard O, Matthysen E. 2015. Niche conservatism among non-native vertebrates in Europe and north America. Ecography 38(3), 321-329.

Suárez-Mota ME, Villaseñor JL, Lápez-mata L. 2015. Ecological niche similarity between congeneric Mexican plant species. Plant ecology and evolution, 148 (3): 318-328.

Thuiller W, Lavorel S, Sykes MT, Araujo MB. 2006. Using niche-based modeling to assess the impact of climate change on tree functional diversity in Europe. Diversity and Distribution 12 (1), 49-60.

Vale VG, Tarroso P, Brito JC. 2014. Predicting species distribution at range margins: testing the effects of study area extent, resolution and threshold selection in the Sahara-Sahel transition zone. Diversity and Distribution 20(20), 20-33).

Vea IM, Gwiazdowski RA, Normark BB. 2012. Corroborating molecular species discovery: four new pinefeeding species of Chionaspis (Hemiptera, Diaspididae). Zookeys 270, 37-58.

Via S. 2001. Sympatric speciation in animals: the ugly ducking grows up. Trends in Ecology and Evolution 16, 381-390.

Waltari E, Hickerson MJ. 2012. Late Pleistocene species distribution modeling of North Atlantic interdidal invertebrates. Journal of Biogeography 40(2), 249-260.

Warren DL, Glor RE, Turelli M. 2008. Environmental niche equivalency versus conservatism: quantitative approaches to niche evolution. Evolution 62, 2868-2883.

Warren DL, Glor RE, Turelli M. 2010. ENMTools: a toolbox for comparative studies of environmental niche models. Ecography 33, 607-611.

Warren DL, Seifert SN. 2011. Ecological niche modeling in Maxent: the importance of model complexity and the performance of model selection criteria. Ecological applications 21(2), 335-342.

Wellenreuther M, Larson KW, Svensson EI. 2012. Climatic niche divergence or onservatism? Environmental niches and range limits in ecologically similar damselflies. Ecology 93, 1352-1366. 
511 Wei JF, Zhao Q, Zhao WQ, Zhang HF. 2018. Predicting the potential distributions of the invasive cycad 512 scale Aulacaspis yasumatsui (Hemiptera, Diaspididae) under different climate change scenarios and the 513 implications for management. Peer $J$ 6, 4832.

514 Wiens J. 2004. Speciation and ecology revisited: phylogenetic niche conservatism and the origin of species. 515 Evolution 58, 193-197.

516 Wisz MS, Hijmans RJ, Li J, Peterson AT, Graham CH, Guisan A. 2008. Effects of sample size on the 517 performance of species distribution models. Diversity and distributions 14(5), 763-773.

518 Zheng HL, Fang LQ, Milne RI, Zhang L, Wang YL, Mao KS. 2017. Species delimitation and lineage 519 separation history of a species complex of Aspens in China. Front of Plant Science B 8(375), 1-17. 
Figure 1

Study areas and distribution locations for seven cryptic species in geographical space.

The figure show a geographic minimum convex polygon. Different symbols represent different species. The base map was created with Natural Earth Dataset (Http://www.naturalearthdata.com).

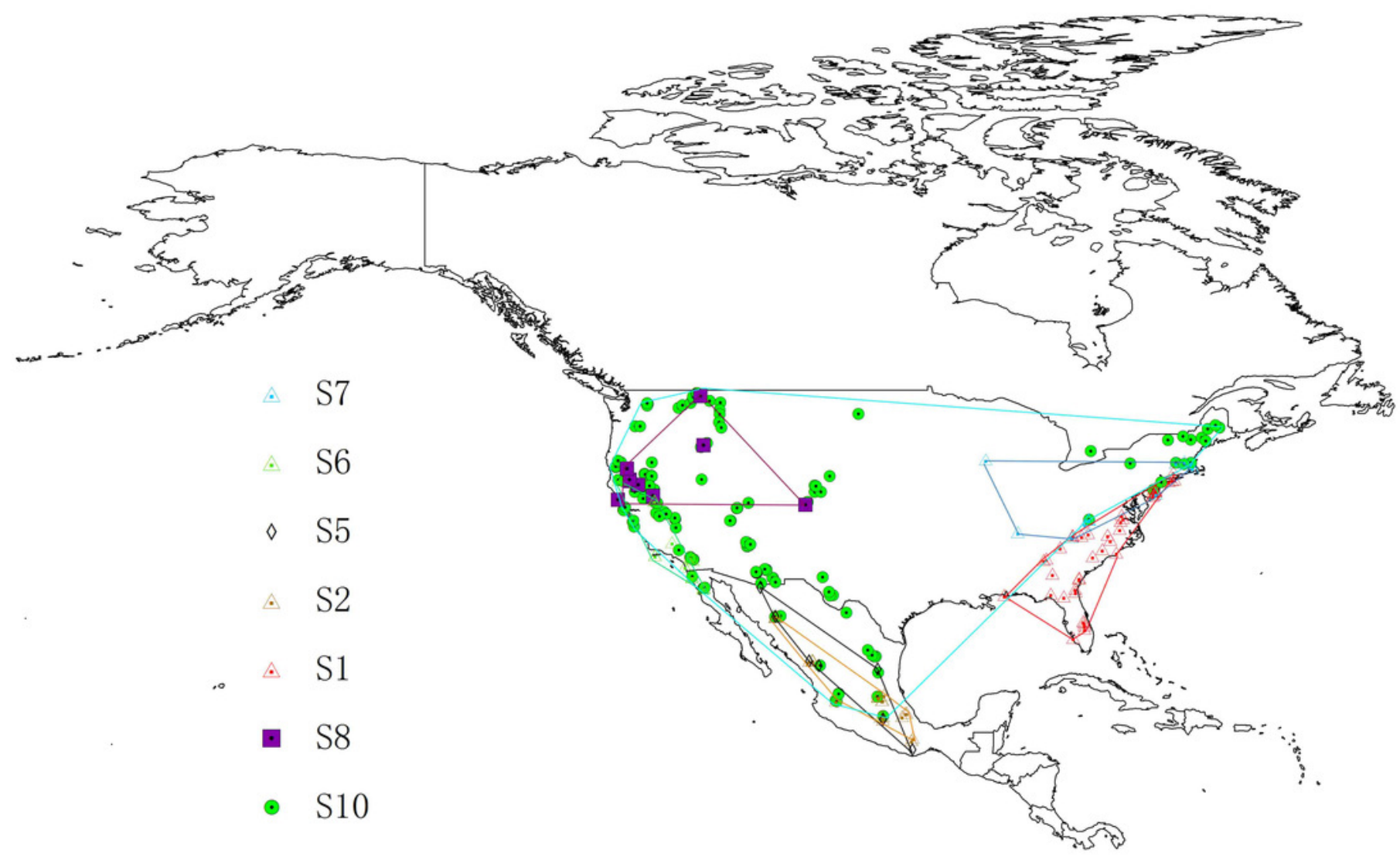


Figure 2

The ecological response curves for each cryptic species.

The response curves are based on the ENMs. Response curves show the ranges in environmental conditions that are more favourable for the distribution of the species. The $x$ axis of the variables represents their ranges for the complete study area, while the $y$-axis represents the predicted suitability of focus species.

Bio2

Bio8

Bio9

Bio12

Bio18

Bio19
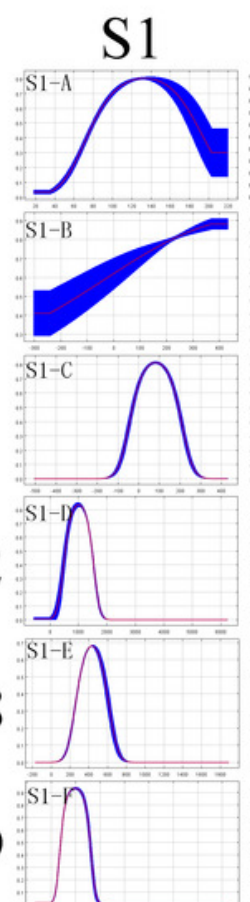
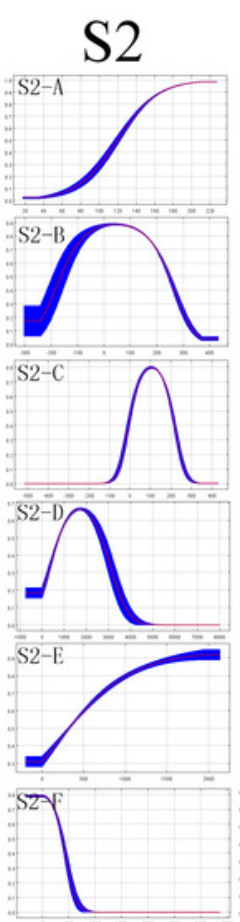

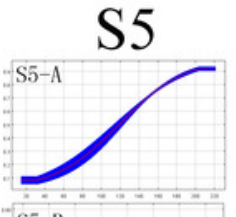

S5-B
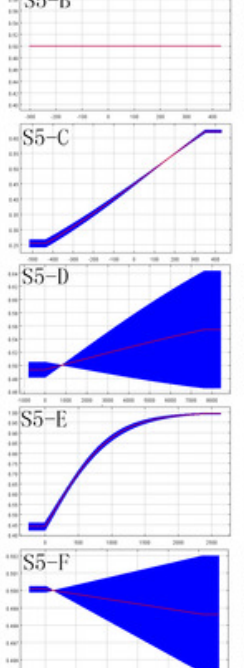
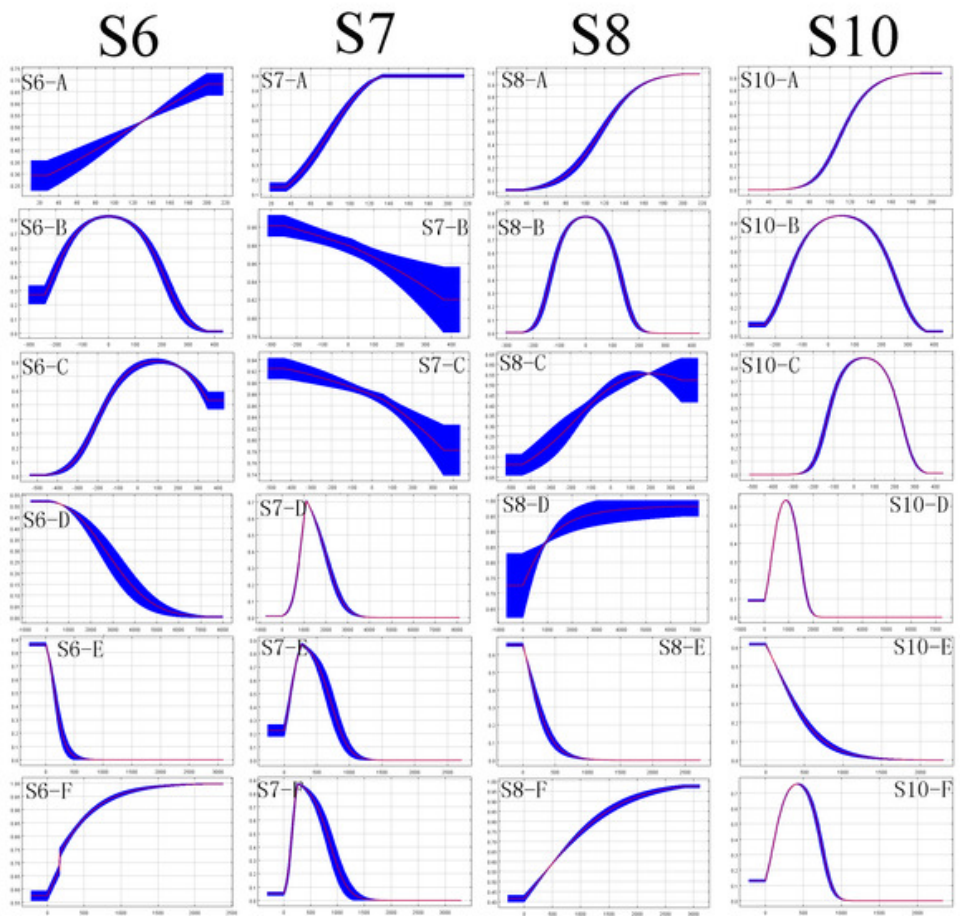


\section{Figure 3}

Potential distributions of each cryptic species

For panels (a-g) represents the areas that have high suitability after applying the LPT (minimum training presence threshold). Panels (a) S1, (b) S2, (c) S5, (d) S6, (e) S7, (f) S8, (g) S10. Niche models results were modelfied in ArcGis 10.1 (Environmental Systems Research Institute, www.esti.com). The base map was created with Natural Earth Dataset (Http://www.naturalearthdata.com) 
(a)

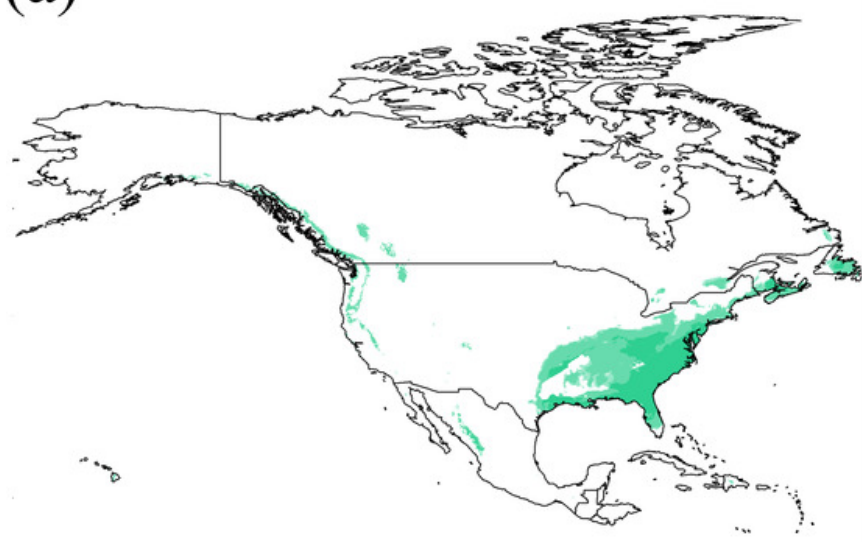

(c)
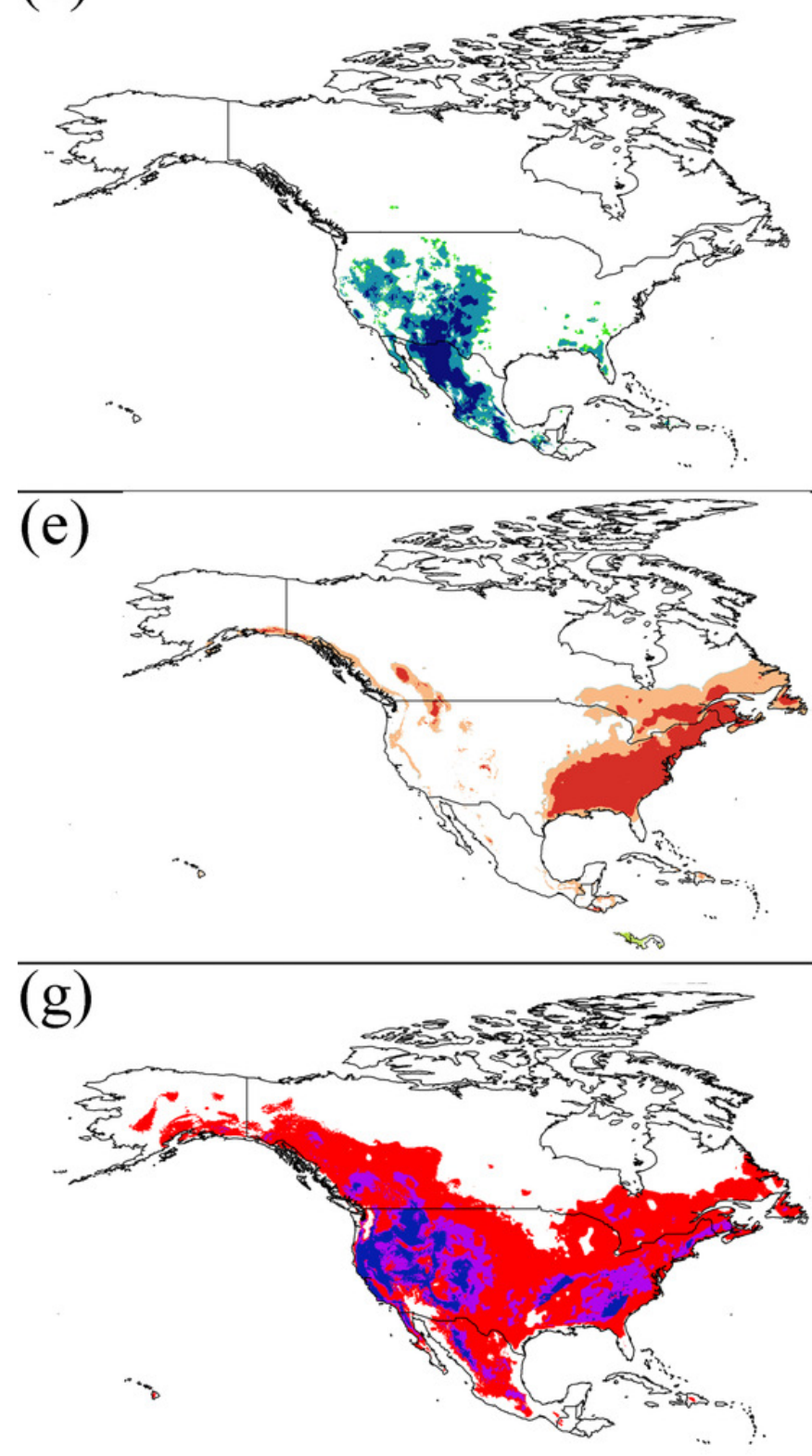

(b)

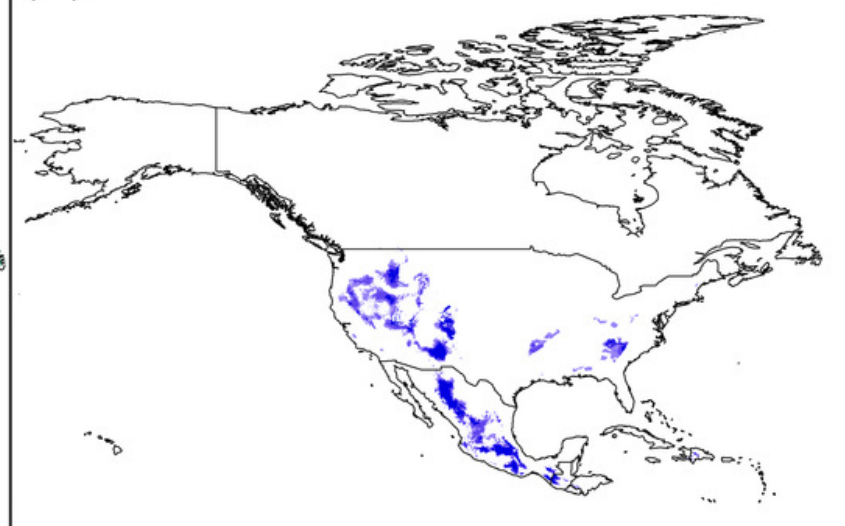

(d)

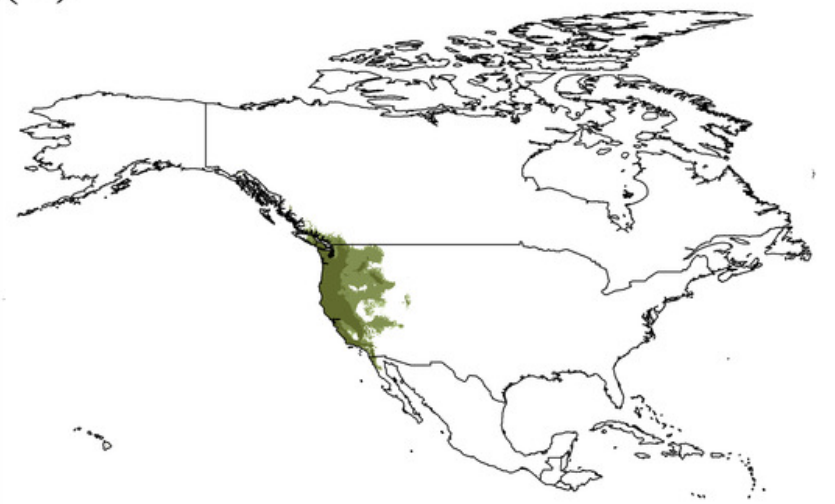

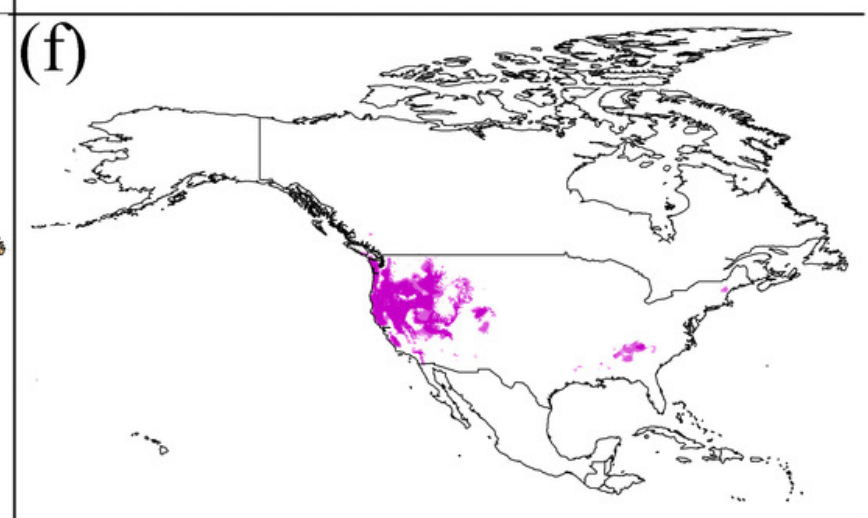




\section{Table 1 (on next page)}

The LPT threshold value of each model from MaxEnt 
1 Table 1 The LPT threshold value of each model from MaxEnt

2

\begin{tabular}{|l|l|l|l|l|l|l|l|}
\hline Species & S1 & S2 & S5 & S6 & S7 & S8 & S10 \\
\hline Value & 0.1779 & 0.509 & 0.586 & 0.580 & 0.305 & 0.540 & 0.029 \\
\hline
\end{tabular}

3

4 Average LPT $($ Lowest presence threshold $)=$ minimum training presence threshold 5

6 


\section{Table 2 (on next page)}

Percentage of variable contribution to the model construction, derived from the permutation importance analysis from MaxEnt.

For each armored scale insect taxon, the two variables with highest contributions are presented in bold 
1 Table 2 Percentage of variable contribution to the model construction, derived from the permutation importance analysis from MaxEnt.

2 (For each armored scale insect taxon, the two variables with highest contributions are presented in bold)

\begin{tabular}{lccccccc}
\hline \multicolumn{1}{c}{ Variable } & $\mathrm{S} 1$ & $\mathrm{~S} 2$ & $\mathrm{~S} 5$ & $\mathrm{~S} 6$ & $\mathrm{~S} 7$ & $\mathrm{~S} 8$ & $\mathrm{~S} 10$ \\
\hline Mean diurnal range (Bio2) & 6.3 & $\mathbf{3 3 . 7}$ & $\mathbf{6 5 . 8}$ & 1 & $\mathbf{1 7 . 4}$ & $\mathbf{3 5 . 2}$ & $\mathbf{3 6 . 7}$ \\
Mean temperature of wettest quarter (Bio8) & 1.8 & 7.9 & 0.2 & $\mathbf{4 3 . 3}$ & 2.8 & $\mathbf{4 7 . 3}$ & $\mathbf{2 3}$ \\
Mean temperature of driest quarter (Bio9) & $\mathbf{3 0 . 6}$ & $\mathbf{3 3 . 6}$ & 3.5 & $\mathbf{3 4}$ & 4.6 & 5.8 & 17.6 \\
Annual precipitation (Bio12) & 20.3 & 5.9 & 0 & 0 & 1.5 & 0.3 & 2.1 \\
Precipitation of warmest quarter (Bio18) & 8 & 14.5 & $\mathbf{3 0 . 5}$ & 15.8 & 15.8 & 0.1 & 0.1 \\
Precipitation of coldest quarter (Bio19) & $\mathbf{3 3}$ & 4.3 & 0 & 6 & $\mathbf{5 8}$ & 11.2 & 20.4 \\
\hline
\end{tabular}




\section{Table 3(on next page)}

Niche comparisons for the cryptic species complex

Niche overlap values are presented for the comparisons of niche similarity and equivalency of species 1 with species 2 . All of the comparisons between the species highlight the nonequivalency of their niche. 
1 Table 3 Niche comparisons for the cryptic species complex. Niche overlap values are presented for the comparisons of

2 niche similarity and equivalency of species 1 with species 2 . All of the comparisons between the species highlight the 3 nonequivalency of their niche.

4

\begin{tabular}{|c|c|c|c|c|}
\hline \multicolumn{2}{|c|}{ Species } & & \multicolumn{2}{c|}{ Niche similarity } \\
\hline 1 & 2 & Niche overlap (D) & $1 \rightarrow 2$ & $1 \leftarrow 2$ \\
\hline S1 & S2 & 0.36 & Similar* & Similar* \\
\hline & S5 & 0.476 & Similar* & Similar* \\
\hline & S6 & 0.014 & ns & ns \\
\hline & S7 & 0.0421 & Similar* & Similar* \\
\hline & S8 & 0.142 & ns & ns \\
\hline & S10 & 0.231 & Similar* & ns \\
\hline S2 & S5 & 0.488 & Similar* & Similar* \\
\hline & S6 & 0.097 & ns & ns \\
\hline & S7 & 0.477 & Similar* & Similar* \\
\hline & S8 & 0.0146 & ns & ns \\
\hline & S10 & 0.351 & Similar* & Similar* \\
\hline S5 & S6 & 0.158 & ns & Similar* \\
\hline & S7 & 0.39 & ns & Similar* \\
\hline & S8 & 0.345 & Similar* & Similar* \\
\hline & S10 & 0.325 & Similar* & Similar* \\
\hline S6 & S7 & 0.246 & Similar* & Similar* \\
\hline & S8 & 0.649 & Similar* & Similar* \\
\hline & S10 & 0.37 & Similar* & Similar* \\
\hline S7 & S8 & 0.477 & Similar* & Similar* \\
\hline & S10 & 0.437 & Similar* & Similar* \\
\hline S8 & S10 & 0.325 & ns & Similar* \\
\hline
\end{tabular}

5

6 ns: no significantly different 